\title{
Evaluation of semen quality brought from Kundasale as replacement of locally produced semen for artificial insemination in Jaffna Peninsula, Sri Lanka
}

\author{
Nilani $\mathrm{K}^{1}$, Eswaramohan $\mathrm{T}^{1}$, Mahadhevan $\mathrm{P}^{2}$ \\ ${ }^{1}$ Department of Zoology, Faculty of Science, University of Jaffna. \\ ${ }^{2}$ Artificial Insemination Centre, Thirunelvely, Sri Lanka.
}

Received : 06 November $2012 \quad$ In Final Form: 14 April 2013

\begin{abstract}
Artificial Insemination (Al) with frozen semen has been proved as the most potent method for rapid genetic improvement in domestic animals. While the use of Al with fresh semen is limited by the restricted period of sperm viability, this limitation may be overcome by the storage of frozen semen. Recently Al center of Thirunelvely is being advised to use the deep-frozen semen processed from Kundasale of Central Province (CP) for Al purposes. Thus the present study was undertaken to compare the quality of locally produced stored semen (LPS) and semen brought from Kundasale in deep-frozen condition (KDFS) by assessing the viability of them as the prominent factor that determine the successful of $A \mathrm{I}$ service. A batch of LPS and KDFS was obtained from the Al center at Thirunelvely. LPS was diluted with $2.9 \%$ sodium citrate buffer solution to evaluate the individual progressive motility and sperm velocity, sperm morphology, and acrosome integrity. Sperm smear was prepared by using $1 \%$ eosin and $0.75 \%$ Giemsa stain to identify intact and reacted acrosome and sperm abnormalities respectively. Thawed semen was also assessed for motility examination, percentage of intact acrosome, viability and morphology. Paired' $t$ ' test was performed to compare the means of the viability of LPS and KDFS $(P<0.05)$. Even though the viability and sperm velocity of KDFS $(84.57 \pm 0.9957 \%, 24.75 \pm 1.887 \mu \mathrm{m} / \mathrm{s})$ was lower than LPS $(96.62 \pm 0.7837 \%, 26.00 \pm 1.581 \mu \mathrm{m} / \mathrm{s})$, its viability is enough for successful conception rate. Therefore usage of KDFS instead of LPS won't affect the successful rate of Al service in Jaffna Peninsula.
\end{abstract}

Key words: Cryopreservation, Thawed semen, acrosome status

\section{Introduction}

Artificial Insemination (Al) with frozen semen has been proved as the most potent method for rapid genetic improvement in domestic animals. The advantages of Al have been well

"Department of Zoology, Faculty of Science, University of Jaffna, Sri Lanka. 
recognized in the dairy industry where highly selected and genetically superior bulls are used [1]. While the use of Al with fresh semen is limited by the restricted period of sperm viability, this limitation may be overcome by the storage of frozen semen. At present, low fertility associated with high embryonic mortality is a consequence of the use of frozen semen. The search for a successful cryopreservation protocol for semen can be traced back 200 years to the discovery that human, stallion, and frog spermatozoa rendered inactive by cooling in snow could be revived [2]. Although the basic principle of cryopreservation is similar for spermatozoa of most mammalian species, the sperm from different species may react differently to freezing due to their difference in morphology and certain biochemical constituents. Therefore, a cryopreservation protocol developed for one species may not be ideal for sperm of other species [3]. Cryopreserved semen management for artificial insemination is a crucial step towards obtaining acceptable pregnancy rates. Thereby the main goal of semen evaluation is to predict its fertilizing ability [4] and several cell staining techniques have been developed to evaluate sperm damages caused by cryopreservation, such as the eosinnigrosin staining. Nonetheless, the surviving sperm population might have morphological defects that reduce and impairs their fertilizing ability, and it is well known that cryopreserved semen presents sub lethal damages and fertility reduction [5]. Although $\mathrm{Al}$ with locally produced stored semen (LPS) has been performed at Al center, Thirunelvely in Jaffna peninsula since 1957, recently this Al center is being advised to use the deep-frozen semen processed from Kundasale (KDFS) of CP for Al purposes. Thus the present study was undertaken to compare the quality of LPS and KDFS by assessing the viability of them as the prominent factor that determine the successful of Al service.

\section{MATERIAL AND METHODS}

\section{Evaluation of locally produced stored semen (LPS)}

A batch of LPS and KDFS was obtained from the Al center at Thirunelvely. LPS was diluted with $2.9 \%$ sodium citrate buffer solution to evaluate the individual progressive motility and sperm velocity under the light microscope (10X objective) by using hemocytometer and stopwatch. Sperm smear was prepared by using $1 \%$ eosin and $0.75 \%$ Giemsa stain to identify intact and reacted acrosome, abnormal heads, proximal and distal cytoplasmic droplets, abnormal 
middle piece, double tails, double heads, and crooked tails, etc.

Evaluation of deep-frozen semen processed from Kundasale (KDFS)

The thawing of straws $\left(-180^{\circ} \mathrm{C}\right)$ in the liquid N2 of storage container was performed by using the water bath at the temperature of $37^{\circ} \mathrm{C}$ for $30-40$ seconds. Then one end of the straw was cut to empty the contents into eppendorf tube. Thawed semen was diluted with 2.9\% sodium citrate buffer solution for further analysis.

\section{Motility examination of KDFS}

The percentage progressive motility was determined by visual observation immediately after thawing the semen. A drop of semen was spread thinly and evenly between a warm slide and cover slip at a magnification of 1000 (with oil immersion) to evaluate the percentage of spermatozoa with progressive motility, and then the rate of progression was assessed on the following scale:

$0=$ No movement

1 = Slight ripple or vibration at the tail, without progression

2 = Slow progression, including stop and start movements
$3=$ Continuous progressive movement at a moderate speed

$4=$ Progressive and rapid movement

$5=$ Very fast progressive movement, with the cells being difficult to follow visually

\section{Percentage of intact acrosome}

The Spermatozoa were immobilized by addition of formaldehyde $(37 \% \mathrm{w} / \mathrm{v})$ commercial solution to examine the acrosome integrity (6). The semen was diluted to make 1:200 dilutions and then it was stained with $0.75 \%$ Giemsa for overnight. The number of sperms with intact and reacted acrosome was counted under light microscope (40X objective).

\section{Viability and morphology}

The determination of these parameters was done using same methodology as for LPS. Sperm viability and sperm abnormality was determined by microscopic observation of a smear of semen subjected to eosin staining under the light microscope at X1000 magnification. A total of 200 sperms were counted and the percentage of nonviable cells, abnormal acrosome and malformations of the sperm were calculated. A whole set of experiment was conducted as triplicate. 


\section{Statistical analysis}

Paired ' $\mathrm{t}$ ' test by using prism 5.04 was performed to compare the viability and sperm velocity of LPS and deep-frozen semen.

\section{RESULTS AND DISCUSSION}

The present study demonstrated that the viability of KDFS was slightly lower than LPS (Fig 1) whereas the average progressive individual motility was greater than $24 \mu \mathrm{m} / \mathrm{s}$ which is slightly lower than LPS (Table 1), because
Exceptionally some sperms in thawed semen moved very faster than in LPS (>29 $\mu \mathrm{m} / \mathrm{s}$ ), but reason for this is inconclusive. After eosins staining dead sperms (Fig 2A) were easily identified as they absorbed the stain and appeared as red in colour, but live sperms (Fig 2B) don't absorb the stain.

Percentage of sperms with intact acrosome (Fig 2C) was approximately $90 \%$. This percentage is low compared to the LPS (98\%) due to the reaction of the acrosome (Fig 2D) during the freezing

Table 1: Comparison of the viability and sperm velocity of fresh and frozen-thawed semen.

\begin{tabular}{|c|c|c|}
\hline Semen samples & Viability (\%) & Sperm velocity $(\mu \mathrm{m} / \mathrm{s})$ \\
\hline LPS & $96.62 \pm 0.7837^{*}$ & $26.00 \pm 1.581$ \\
\hline KDFS & $84.57 \pm 0.9957^{*}$ & $24.75 \pm 1.887$ \\
\hline
\end{tabular}
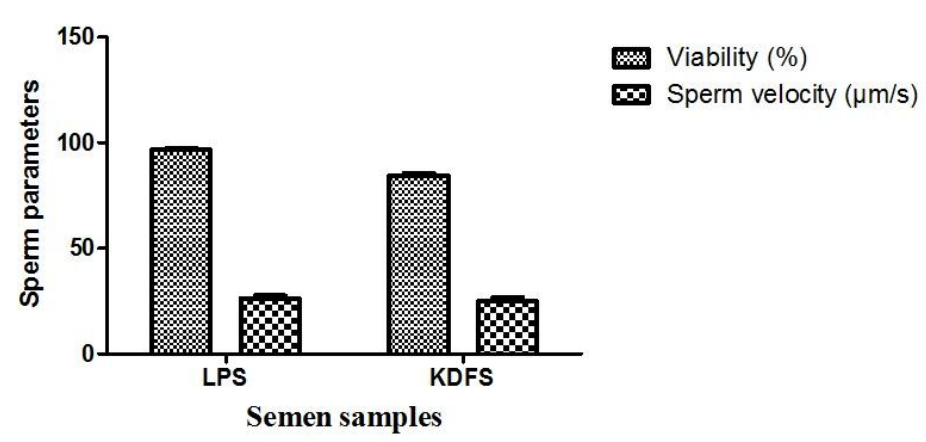

Fig 1: Comparison of the viability and sperm velocity of LPS and KDFS bull semen.

freezing and thawing may reduce progressive motility, membrane integrity and acrosome status which leads to poor fertility following cervical insemination. and thawing procedures. In our studies sperm abnormalities of both samples including coiled tail (Fig 2E), double headed sperm (Fig 2F), coiled mid piece 
(Fig 2G), detached head (Fig 2H) were lower than $15 \%$.

The efficient preservation of sperm cells with full fertilization capacity is the main objective of the semen cryopreservation process. Generally semen is stored in liquid nitrogen, because its ability to maintain temperatures far below the freezing point of water. At each stage of the cryopreservation cycle, which includes the entire process of semen collection, dilution, equilibration and freezing, the spermatozoa may lose the ability to fertilize normally [7]. of acrosome integrity [9] and although, glycerol is commonly used as an intracellular cryoprotectant, it exerts osmotic and toxic effects on the cryopreserved cells, to the point of been contraceptive for sperm of many species $[10,11,12,13,14]$. The degree of damage may depend on the composition of the semen extenders and the nature of the cryoprotectant. Integrity of acrosomal function is of crucial importance to normal fertilization, because acrosomeless, membrane damaged and cryocapacitated sperm cannot fertilize oocytes $[8,15,16]$.

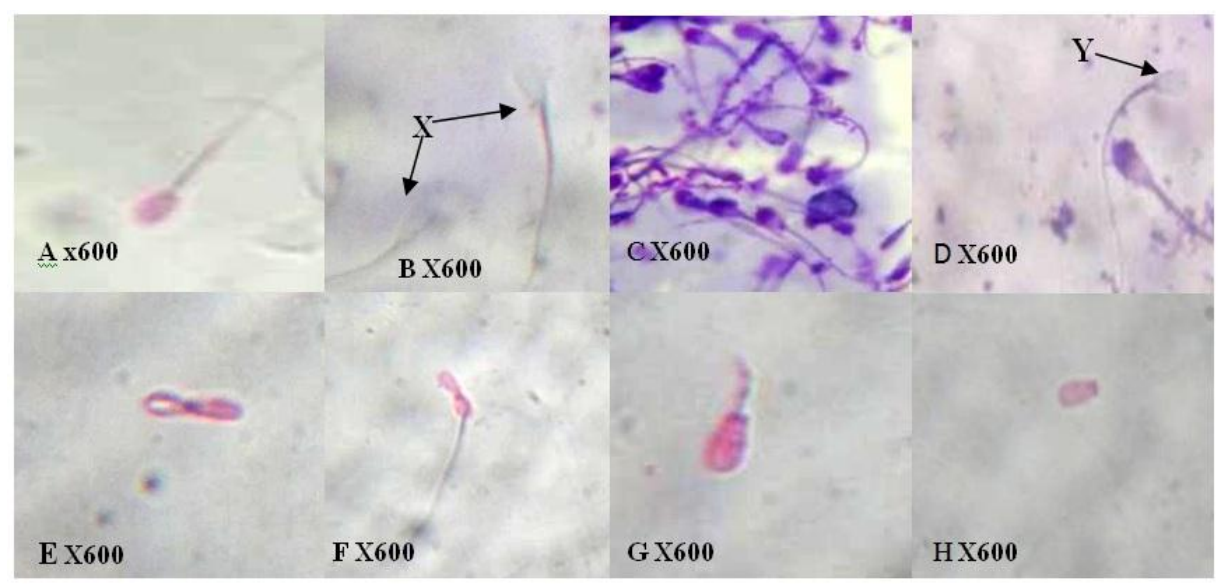

Fig 2: Assessment of the acrosome integrity (0.75\% Giemsa stain), sperm membrane and sperm abnormalities (1\% eosin) under the light microscope A: dead sperm B: live sperms $(X)$ $C$ : acrosome intact sperms $D$ : acrosome intact sperms and acrosome reacted sperm (Y) E: coiled tail F: double headed sperm G: coiled midpiece H: detached head.

The main cause of cell damage during cryopreservation is attributed to intracellular ice formation [8], producing sperm membrane deterioration and loss
Therefore, it is important to analyze frozen-thawed semen for sperm viability and simultaneously determine acrosome integrity [17]. 
Determining the percentage of intact acrosomes is a morphological method for measuring the viability after thawing, and is related to fertility. Recently, the level of acrosome reacted sperm exposed to cryopreservation has been used to estimate sperm function as a response to cryopreservation and several assays have been developed to detect morphological and functional integrity of plasma membrane and sperm acrosomal membrane $[16,18,19,20,21]$.

The discovery that egg yolk protected spermatozoa against cold shock [22] led to the commercial development of $\mathrm{Al}$ in cattle. Protective agents such as egg yolk are of value even when spermatozoa are not subject to cold shock because seminal plasma is known to have a detrimental effect on the survival of spermatozoa in diluted bovine semen $[23,24,25,26]$.

Large changes in semen $\mathrm{pH}$ can result in sperm damage, infertility, or sperm mortality. Therefore, in order to sustain the viability and fertilizing ability of sperm it is essential to maintain a proper environment by controlling the $\mathrm{pH}$ fluctuations in the cryopreservation media.

\section{CONCLUSION}

Even though the viability and sperm velocity of cryopreserved semen was lower than fresh semen, its viability is enough for successful conception rate. Therefore usage of KDFS instead of LPS won't affect the successful rate of $\mathrm{Al}$ service in Jaffna Peninsula.

\section{ACKNOWLEDGEMENTS}

We would like to thank Mr.S.Senthilrajah, Mr.S.Senthuran, and other staffs of Artificial insemination centre, Thirunelvely, Jaffna for their assistance in handling of semen.

\section{REFERENCES}

1. G. A. Langford, G. J. Marcus, A. J. Hackett, L. Ainsworth, M. S. Wolynetz, and H. F. Peters, Can. J. Anim. Sci. 59, 685-691 (1979)

2. P.F. Watson, In: Finn CA, ed. Oxf. Rev. Reprod. Biol. Oxford, UK: Clarendon Press.1, 283-350 (1979).

3. M.N. Sundararaman and M.J. Edwin, Asian. J. Cell. Biol. 3, 22-33 (2008).

4. F.C. Britto, A.D. Barth, S. BilodeauGoeseels, P.L. Panich, and P. Kastelic, Theriogenology. 60, 1539-1551 (2003).

5. L.M. Correa, A. Thomas, S.A. Meyers, Biol. Reprod. Article in press, 77(6), 942-953 (2007).

6. K. Nilani, T. Eswaramohan and K. Balasubramaniam, IJSRP. 2, 1-5 (2012).

7. P.F. Watson, Reprod. Fertil. Dev.7, 871-891 (1995). 
8. P. Mazur, Am. J. Physiol. 247, 125-142 (1984).

9. F. Szasz, S. Sirivaidyapong, F.P. Chen, W.F. Voorhout, A. Marks, B. Colenbrander, L. Solti, B.M. Gadella, Mol. Reprod. Dev. 55, 289-298 (2000).

10. R.H. Hammerstedt, J.K. Graham, J.P. Nolan, J. Androl. 11, 73-88 (1990).

11. R.H. Hammerstedt, J.K. Graham, Cryobiology 29, 26-38 (1992).

12. M.M. Buhr, P. Fiser, J.L. Bailey, E.F. Curtis, J. Androl. 22, 961-969 (2001).

13. R.S. Jeyendran, H.H. Van-Der-Ven, M. Pérez-Pelaez, L.J. Zaneveld, Andrología. 17, 241-248 (1985).

14. K. Wundrich, V. Paasch, M. Leicht, H.J. Glander, Cell Tissue Bank. 7, 81-90 (2006).

15. L.J. Huo, X.A. Ma, Z.M. Yang, Theriogenology. $\quad 58, \quad 1349-1360$ (2002).

A. Januskauskas, A. Jahannisson, L.K. Soderquist, H. Rodríguez- Martínez, Theriogenology. 53, 859-875 (2000).

16. C.A. Thomas, D.L. Garner, J.M. Dejarnette, C.E. Marshall, Biol. Reprod. 56, 991-998 (1997).
17. A.M. Petrunkina, B. Gröpper, E. Töpfer-Petersen, A.R. Günzel-Apel, Theriogenology. 63, 1390-1406 (2004).

18. S.A Troup, B.A. Lieberman, and P.L. Matson, Hum. Reprod. 9:2079-2073 (1994).

19. A.I. Peña, L.L. Luguilde, M. Barrio, P.G. Herradon, L.A. Quintela, Theriogenology. 59, 1725-1739 (2003).

20. R. Rathi, B. Colenbrander, M.M. Bevers, B.M. Gadela, Biol. Reprod. 65, 462-470, (2001).

21. P.H. Philips, J. biol. Chem. 130, 415 (1939).

22. P. Shannon, J. Dairy Sci. 48, 1357-1361 (1965b).

23. P. Shannon, J. Dairy Sci. 48, 1362-1365 (1965c).

24. J.P. Bennett, and H.M. Dott, Reprod. Fert. 12, 327-336 (1966).

25. H.M. Dott, Reprod. Fert. 38, 147-156 (1974). 